\title{
Estrogen downregulates TAK1 expression in human fibroblast-like synoviocytes and in a rheumatoid arthritis model
}

\author{
$\mathrm{XI} \mathrm{LI}^{1}$ and MIAO $\mathrm{LI}^{2}$ \\ ${ }^{1}$ Department of Sports Medicine and Joint Surgery, The People's Hospital of China Medical University, \\ Shenyang, Liaoning 110000; ${ }^{2}$ Department of Microbiology and Parasitology, College of Basic \\ Medical Sciences, China Medical University, Shenyang, Liaoning 110122, P.R. China
}

Received July 4, 2019; Accepted May 15, 2020

DOI: $10.3892 / \mathrm{etm} .2020 .8848$

\begin{abstract}
Transforming growth factor $\beta$-activated kinase-1 (TAK1), a member of the mitogen-activated protein kinase family, plays a key role in the pathogenesis and progression of rheumatoid arthritis (RA). Estrogen has been previously reported to delay arthritis progression. However, the exact association between TAK1 and estrogen remains elusive. The present study demonstrated that TAK1 was upregulated in synoviocytes of patients with RA compared with patients with osteoarthritis and healthy controls. In addition, TAK1 was also expressed in cultured fibroblast-like synoviocytes (FLS), and its levels decreased significantly in 17 $\beta$-estradiol (E2)-treated cells in a dose-dependent manner. Furthermore, administration of E2 significantly decreased TAK1 expression and attenuated the development of collagen-induced arthritis (CIA). Taken together, the findings of the present study suggested that E2 mediates a decrease of TAK1 in both FLS and CIA, which subsequently results in a suppression of the pathological process of CIA. Therefore, estrogen may serve as a potential therapeutic agent for the treatment of RA by targeting TAK1.
\end{abstract}

\section{Introduction}

Human rheumatoid arthritis (RA) is characterized by chronic inflammation and destruction of multiple joints (1). Fibroblast-like synoviocytes (FLS) in synovial tissues play critical roles in the pathogenesis of RA, such as initiation of inflammatory responses and inflammation-associated cartilage damage (2). In addition, FLS can promote various processes in RA by secreting different types of inflamma-

Correspondence to: Professor Miao Li, Department of Microbiology and Parasitology, College of Basic Medical Sciences, China Medical University, 77 Puhe Road, Shenyang North New Area, Shenyang, Liaoning 110122, P.R. China

E-mail: sendtolm@126.com

Key words: transforming growth factor $\beta$-activated kinase-1, 17 $\beta$-estradiol, rheumatoid arthritis, collagen-induced arthritis, fibroblast-like synoviocytes tory cytokines, which has been demonstrated to be closely associated with the activation of cellular mitogen-activated protein kinase (MAPK) and nuclear factor (NF) $-\kappa B$ signaling pathways (3-5). Transforming growth factor $\beta$-activated kinase-1 (TAK1) is a member of the MAPK family. A recent study has reported that TAK1 is involved in cartilage and bone destruction through its downstream signaling molecules, which are involved in pro-inflammatory cytokine signaling, including c-Jun N-terminal kinase (JNK), p38 and NF-кB (6). Choi et al (7) demonstrated that TAK1, a dominant MAP3K, is involved in JNK activation in RA-FLS. Knockdown of TAK1 in RA-FLS effectively inhibits the activation of interleukin (IL)-1 $\beta$-induced activator protein 1 and matrix metalloproteinase (MMP)3 expression, and IL-6 production. Notably, in RA-FLS, signaling molecules are dependent on TAK1, such as tumor necrosis factor, toll-like receptor (TLR)2 and IL-1, but not TLR4 (8). These findings demonstrated TAK1 to be a central regulator of cytokine signaling networks in RA, and a therapeutic strategy for inhibiting TAK1 may prove to be beneficial for the treatment of RA.

Sex may affect susceptibility to certain autoimmune diseases, such as RA, which has a 3:1 female:male ratio. Sex hormones and their receptors are the basis of sex-related differences in RA activity (9). Estrogen treatment delays arthritis progression, which has been reported in collagen-induced arthritis (CIA), a well-established experimental model $(10,11)$. However, it is difficult to describe the association between estrogen and RA. Since the pathogenesis of RA involves both estrogen and TAK1, it is important to determine whether TAK1 is an estrogen-responsive gene.

The aim of the present study was to investigate the levels of TAK1 in synoviocytes of patients with RA, and determine whether the effect of $17 \beta$-estradiol (E2) on the level of TAK1 mRNA in FLS is dependent on estrogen receptor (ER). The findings of the present study may help improve our understanding of the association between estrogen and TAK1 and determine whether TAK1 may serve as a potential diagnostic and therapeutic target in the treatment of RA.

\section{Materials and methods}

Materials and reagents. E2, ICI 182,780, bovine type II collagen and Freund's Complete Adjuvant were purchased from 
Sigma-Aldrich; Merck KGaA. Mouse monoclonal antibody against TAK1 (1:200, cat. no. sc-7967), mouse anti-GAPDH monoclonal antibody (1:1,000, cat. no. sc-365062) and mouse IgGK light chain binding protein conjugated to horseradish peroxidase $(1: 1,000$, cat. no. sc-516102) were obtained from Santa Cruz Biotechnology, Inc.

Human synovial tissues and FLS. Human synovial tissue specimens were obtained from 6 healthy controls ( 3 men and 3 women, with ages ranging between 40 and 72 years and a mean age of $55.5 \pm 2.6$ years) who had undergone knee arthroscopic inspection, 6 patients with osteoarthritis $(\mathrm{OA} ; 3$ men and 3 women, with ages ranging between 46 and 72 years and a mean age of $53.0 \pm 4.5$ years) and 6 patients with RA ( 3 men and 3 women, with ages ranging between 38 and 68 years and a mean age of $52.5 \pm 2.8$ years) who had undergone joint replacement surgery or synovectomy at the Department of Sports Medicine and Joint Surgery, The First Affiliated Hospital of China Medical University between January 2007 and December 2007. The present study was approved by the Ethics Committee of China Medical University and all patients provided written informed consent. The RA patients fulfilled the 1987 revised criteria or the 2010 criteria for the disease $(12,13)$. FLS were prepared from synovial tissue as previously described (14). All specimens were stored in liquid nitrogen $\left(195.79^{\circ} \mathrm{C}\right)$ prior to use. For functional assays, FLS were cultured in $0.1 \%$ fetal bovine serum (Gibco; Thermo Fisher Scientific, Inc.).

Reverse transcription-quantitative PCR (RT-qPCR) analysis. RNA isolation was performed using the RNeasy Mini Kit (Qiagen, Inc.). The QuantiTect Reverse Transcription kit (Qiagen, Inc.) was used to synthesize complementary DNA under the following conditions: $42^{\circ} \mathrm{C}$ for $15 \mathrm{~min}$ and $95^{\circ} \mathrm{C}$ for 3 min. qPCR was performed using $\mathrm{RT}^{2} \mathrm{SYBR}^{\circledR}$ Green qPCR Master Mix (cat. no. 330500; Qiagen, Inc.). Samples were normalized to internal control GAPDH and compared to the control group. The primers used in the present study were as follows: TAK1 forward, 5'-ATCGTCATATCAGGCAACG GAC-3' and reverse, 5'-TGAGGTTGGTCCTGA GGTAGT-3'; GAPDH forward, 5'-GCACCGTCAAGGCTGA GAAC-3' and reverse, 5'-TGGTGAAGACGCCAGTGGA-3'. The qPCR reaction conditions were as follows: $10 \mathrm{~min}$ at $95^{\circ} \mathrm{C}$, followed by 40 cycles of $15 \mathrm{sec}$ at $95^{\circ} \mathrm{C}$ and $1 \mathrm{~min}$ at $60^{\circ} \mathrm{C}$. Gene expression values were calculated using the $2^{-\Delta \Delta \mathrm{Cq}}$ relative quantification method, wherein the control group was designated as the calibrator (15).

Western blot analysis. All proteins were isolated using RIPA buffer (Beijing Solarbio Science \& Technology Co., Ltd.) from E2-treated and untreated FLS with a bicinchoninic acid assay to measure protein concentration. The whole-cell extracts (20 mg protein) were fractionated by $10 \%$ SDS-PAGE and blotted onto PVDF membranes. Following blocking of non-specific protein binding in 5\% skimmed milk at room temperature for $1 \mathrm{~h}$, the membranes were incubated at $25^{\circ} \mathrm{C}$ for $4 \mathrm{~h}$ with primary antibodies diluted in primary antibody dilution buffer (Beyotime Institute of Biotechnology). Then, the membranes were further incubated with horseradish peroxidase-conjugated secondary antibody at $25^{\circ} \mathrm{C}$ for $2 \mathrm{~h}$.
The membranes were then visualized using enhanced chemiluminescence (Sigma-Aldrich; Merck KGaA).

CIA.CIA was induced as previously described $(10,11)$. Briefly, bovine type II collagen ( $5 \mathrm{mg}$ ) was dissolved in $25 \mathrm{ml}$ acetic acid with a concentration of $0.1 \mathrm{~mol} / \mathrm{l}$, which was prepared as a solution with a concentration of $2 \mathrm{mg} / \mathrm{ml}$, followed by preservation overnight at $4^{\circ} \mathrm{C}$ in a refrigerator. Subsequently, $2.5 \mathrm{ml}$ Freund's Complete Adjuvant was added to prepare the collagen emulsion, which was stored at $4^{\circ} \mathrm{C}$ until use. A total of 30 female rats (4 months old, weighing 80-100 g) were randomly divided into the normal control $(n=10)$, CIA model $(n=10)$ and E2-CIA $(n=10)$ groups. Rats were purchased from Beijing Vital River Laboratory Animal Technology, Co. All animals were housed at $25^{\circ} \mathrm{C}$ with a 12-h light/dark cycle, with a relative humidity of $40-70 \%$, and had free access to food and water. The experimental protocol conformed to the Animal Welfare Act Guide for Use and Care of Laboratory Animals and was approved by the Institutional Animal Care and Use Committee of China Medical University. Rats in the normal control group were injected via the tail vein with $0.1 \mathrm{ml}$ normal saline, and rats in the CIA model and E2-CIA groups received an injection of $0.1 \mathrm{ml}$ collagen emulsion via the tail vein. At 21 days after the model establishment, drug administration was performed, in which $100 \mu \mathrm{g}$ E2 was injected into rats of the E2-CIA group for 3 days, and normal saline was given to rats in the normal control and CIA model groups. The injection dose of E2 was significantly higher compared with endogenous levels, therefore, normal secretions may not be taken into consideration (16). Assessment of arthritis severity was performed using scores between 0 and 3 for each paw, and each rat was given a maximum of 12 points, determined as follows: 1, Swelling or erythema in one joint; 2 , swelling or erythema in two joints; and 3, severe swelling or erythema of more than two joints, or ankylosis of the entire paw (17). Weighing the uteri at termination confirmed successful estrogen treatment. The rats were euthanized with an overdose of pentobarbital $(200 \mathrm{mg} / \mathrm{kg}$ ) and death was confirmed through observing respiratory and cardiac arrest and the absence of any pain response to needle puncture of the extremities.

Statistical analysis. Statistical analyses were performed using SPSS version 16.0 (SPSS, Inc.). Data are presented as the mean and standard deviation. Student's t test was used to compare two groups and one way ANOVA with Dunnett's post hoc test was used to compare multiple groups. $\mathrm{P}<0.05$ was considered to indicate a statistically significant difference.

\section{Results}

TAK1 level is upregulated in synoviocytes of patients with $R A$. To investigate the role of TAK1 in patients with RA, TAK1 expression levels were first determined in synoviocytes from patients with RA, patients with OA and healthy controls. As shown in Fig. 1A and B, compared with OA patients and healthy controls, patients with RA exhibited an increase in TAK1 mRNA and protein levels. This suggests that an increase of TAK 1 expression may be implicated in RA pathogenesis. 
A

B

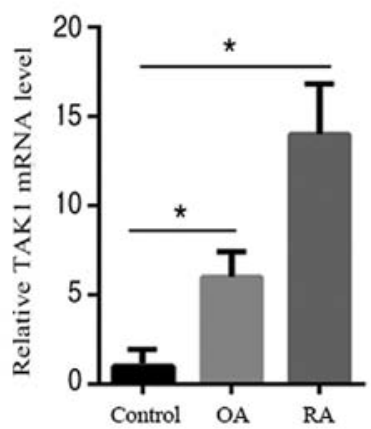

Figure 1. TAK1 expression is increased in FLS from patients with RA. (A) Cells extracted from the synovial tissues of 6 patients with RA, 6 patients with $\mathrm{OA}$ and 6 healthy controls were assessed by reverse transcription-quantitative PCR analysis. The mRNA expression of TAK1 was determined. Data are presented as relative expression units, normalized to GAPDH. Values are the mean \pm standard error of the mean from three independent experiments. ${ }^{*} \mathrm{P}<0.05$. (B) Cells extracted from the synovial tissues of 6 patients with RA, 6 patients with OA and 6 healthy controls were assessed by western blotting. The protein expression of TAK1 was determined. Data are presented as the mean \pm standard error of the mean. RA, rheumatoid arthritis, OA, osteoarthritis; TAK1, transforming growth factor $\beta$-activated kinase-1; FLS, fibroblast-like synoviocytes.

Downregulation of TAK1 expression by E2 in FLS. To assess whether the expression of TAK1 is regulated by E2, the TAK1 mRNA and protein levels were examined in FLS. RT-qPCR analysis demonstrated that TAK1 mRNA expression was evidently decreased by E2 in a dose-dependent manner; compared with the control, mRNA levels decreased by 15,50 and $70 \%$ at E2 concentrations of 1,10 and $100 \mathrm{nM}$, respectively. In addition, concomitant treatment with ICI 182,780, an ER antagonist, restored the inhibition (Fig. 2A). The results of western blot analysis demonstrated that after a 24 h-treatment of FLS with E2 (100 nM), the protein level was also decreased, which is in agreement with the decrease in mRNA (Fig. 2B). These results indicate that $\mathrm{E} 2$ affects the mRNA and protein expression of TAK1 in the genomic pathway involving ER.

Systemic administration of E2 attenuates the severity of CIA by regulating TAK1 gene expression in vivo. The present data suggested that TAK 1 and E2 may both play important roles in the pathogenesis of osteoarthritis in human RA. Therefore, it was next investigated whether E2 could alleviate the inflammation and joint destruction observed in CIA by regulating TAK1 in mice. Mice were randomly assigned to receive treatment with E2 or saline and CIA was established; arthritis development was then assessed. Measurement of swelling degree of the toes at 21 days after model establishment revealed a significant difference in joint swelling degree of the rats' left feet between the CIA model group and the E2-CIA and control groups $(\mathrm{P}<0.01)$, indicating that the models were successfully established. E2 was administered at 21 days after model establishment. The left foot joint swelling degrees of the rats in the normal control, CIA model and E2-CIA groups were detected at 21 days after drug administration. The results demonstrated that there was a significant difference between the E2-CIA and CIA model groups $(\mathrm{P}<0.01$; Table I).
A

B

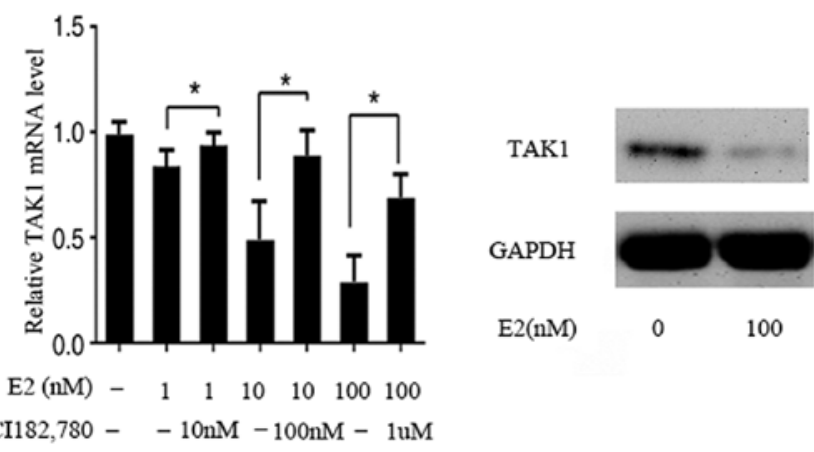

Figure 2. Effect of E2 on TAK1 mRNA and protein expression in FLS (A) FLS were exposed to E2 with or without the ER antagonist ICI 182,780 for $24 \mathrm{~h}$. TAK1 mRNA was measured by reverse transcription-quantitative PCR analysis. Data are presented as relative expression units, normalized to GAPDH. Values are the mean \pm standard error of the mean from three independent experiments. ${ }^{*} \mathrm{P}<0.05$. (B) TAK1 and GAPDH levels were determined by western blot analysis of FLS that were incubated with or without E2 for $24 \mathrm{~h}$. Data are presented as the mean \pm standard error of the mean. TAK1, transforming growth factor $\beta$-activated kinase-1; FLS, fibroblast-like synoviocytes; E2, 17 $\beta$-estradiol.

By using X-ray imaging, the effect pre- and post-administration of E2 on joint swelling and destruction in CIA mice was also observed. The results demonstrated that the soft tissue in the control group was not swollen and the joint remained intact. In the CIA group, the soft tissue was swollen and the tarsal bones were scattered; osteophytes were seen around the tarsal bones, and free 'articular bones' were observed. However, soft tissue swelling and joint damage were significantly relieved in the E2-CIA group following administration of E2 (Fig. 3A).

TAK1 is a well-known key player in the pathogenesis of RA. Therefore, the present study examined whether administration of E2 was associated with the level of TAK1 in vivo. RT-qPCR analysis and western blotting revealed a significant decrease in TAK1 expression following treatment with E2. As shown in Fig. 3B and C, the expression of TAK1 in joint soft tissue in the CIA model group was significantly increased compared with that in the control group $(\mathrm{P}<0.05)$. TAK1 expression was significantly lower in the E2-CIA group compared with that in the CIA group $(\mathrm{P}<0.05)$. In summary, these results suggest that systemic administration of E2 ameliorates the incidence and severity of CIA through regulation of TAK1 expression in mice.

\section{Discussion}

To the best of our knowledge, the present study was the first to report that TAK1 is present in the synoviocytes of patients with RA. In addition, E2 decreased the expression of TAK1 in FLS, an effect that was dependent on ER. Furthermore, systemic administration of E2 markedly reduced the incidence and symptoms of arthritis, and decreased the expression of TAK1 in mice with CIA.

In recent years, a number of studies have suggested that TAK1 is implicated in the pathogenesis of RA. According to an epistasis analysis in a study by Gottar-Guillier et al, as a downstream mediator of TAK1 in the inflammatory signaling pathways, IL- 8 production in primary human endothelial 
Table I. Comparison of joint swelling degrees of the left posterior foot among three groups of rats $(n=10)$.

\begin{tabular}{lccc}
\hline & $\begin{array}{c}\text { Swelling degree of the } \\
\text { left posterior foot after } \\
\text { model establishment }\end{array}$ & & \multicolumn{2}{c}{$\begin{array}{c}\text { Swelling degree of the } \\
\text { left posterior foot after } \\
\text { drug administration }\end{array}$} \\
\cline { 2 - 3 } Groups & 21 days & & 14 days \\
\hline Control & 0 & 0 & 0 \\
CIA & $2.1 \pm 0.2^{\mathrm{a}}$ & $2.5 \pm 0.4^{\mathrm{a}}$ & 0 \\
E2-CIA & $2.0 \pm 0.1^{\mathrm{a}}$ & $1.6 \pm 0.2^{\mathrm{a}, \mathrm{b}}$ & $2.8 \pm 0.2^{\mathrm{a}}$ \\
\hline
\end{tabular}

${ }^{\mathrm{a}} \mathrm{P}<0.01$ vs. control group. ${ }^{\mathrm{b}} \mathrm{P}<0.01$ vs. CIA. CIA, collagen-induced arthritis; E2, $17 \beta$-estradiol.
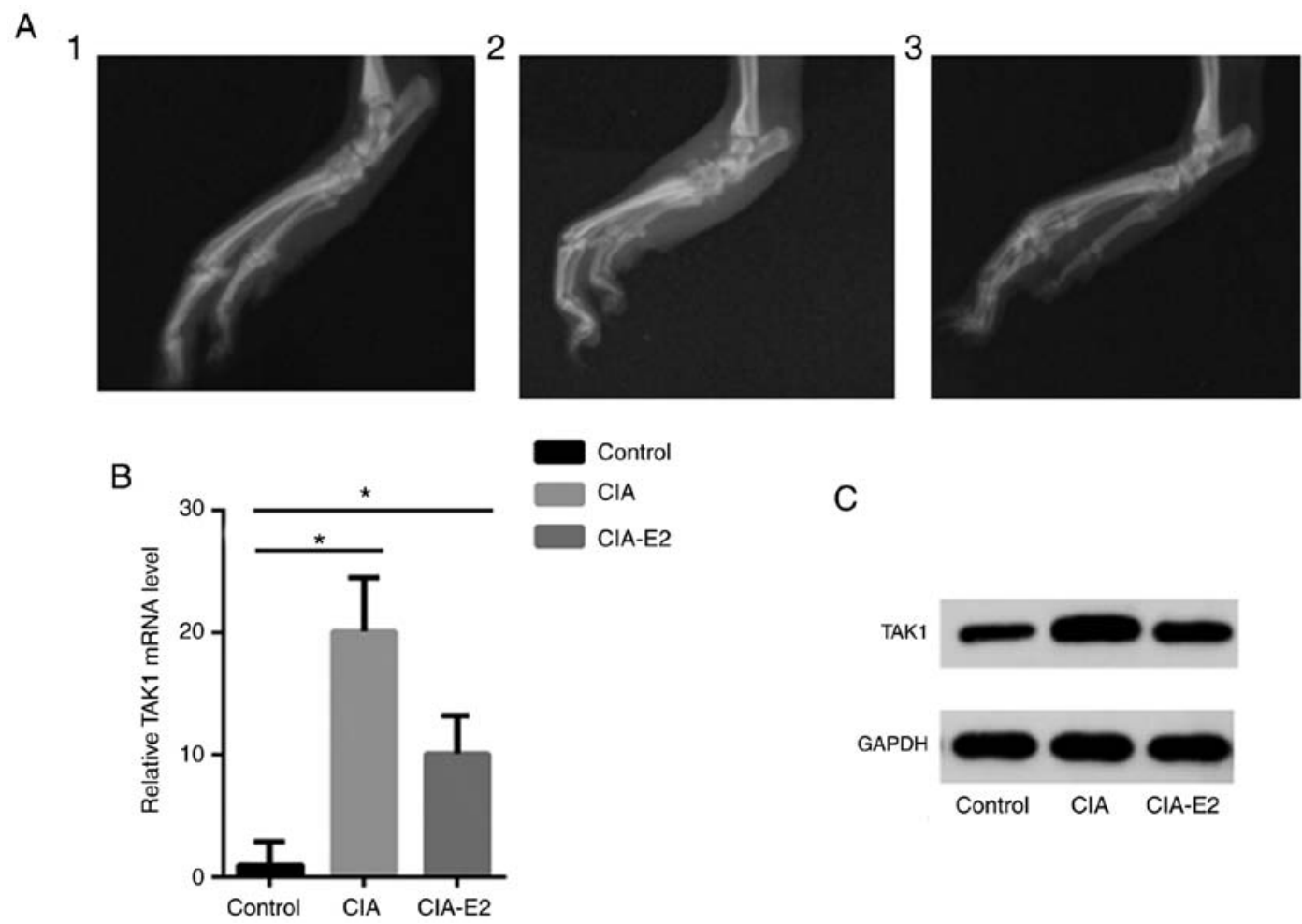

Figure 3. Systemic administration of E2 attenuates the severity of CIA by regulating TAK1 gene expression in vivo. CIA was treated with E2 (100 $\mu \mathrm{g} / \mathrm{day})$ or saline for 3 days. Arthritis severity was assessed by macroscopic scoring of arthritis in the paws. (A) X-ray imaging demonstrates joint damage. 1, control group; 2, CIA group; 3, E2-CIA group. Macroscopic evidence of arthritis, such as swelling or scattered tarsal bones, was markedly observed in the CIA group, while treatment with E2 significantly attenuated arthritis severity in CIA. (B) The mRNA level of TAK1 was determined by reverse transcription-quantitative PCR analysis. Data are presented as relative expression units, normalized to GAPDH. Values are the mean \pm standard error of the mean from three independent experiments. "P<0.05. (C) TAK1 protein expression was determined by western blotting. Data are presented as the mean \pm standard error of the mean. CIA, collagen-induced arthritis; E2, 17 $\beta$-estradiol; TAK1, transforming growth factor $\beta$-activated kinase-1.

cells was inhibited by small interfering RNA knockdown of the tyrosine kinase bone marrow kinase on chromosome $\mathrm{X}$ (BMX). Furthermore, BMX-deficient mice were protected from developing $\mathrm{K} / \mathrm{BxN}$ serum transfer-induced arthritis. This indicates a potential role for TAK1 in RA pathogenesis through BMX possibly working together with the TAK1-TAB1 complex (18).

The peak incidence of RA in women coincides with the perimenopausal period, when estrogen levels rapidly decline (19). During pregnancy, when the levels of sex hormones increase, $\leq 75 \%$ patients with RA experience symptom relief, which suggests an association between estrogen deficiency and the development of RA (20). By contrast, men have fairly continuous levels of estrogen throughout their adult lives (21). In addition, estrogen levels tend to be lower in postmenopausal women compared with those in men of similar ages (22). A major pathological RA manifestation is reorganization of the synovial architecture, with immune cells infiltrating the synovium, FLS proliferation, synovial inflammation and pannus formation (23). Estrogen exerts a direct effect on monocytes and macrophages. It can increase the production of proinflammatory cytokines, which promote cartilage reabsorption, inhibit proteoglycan synthesis and trigger inflammation, which are all characteristic of RA (24-26). However, to date, there is limited evidence on the association between TAK1 and E2. 
In the present study, E2, a terminal active estrogen with the highest affinity for ER, was used to determine the effect of estrogen on TAK1 expression in FLS. It was observed that E2 decreased the mRNA level of TAK1 in a dose-dependent manner, and this decrease was prevented by the selective ER antagonist ICI 182,780 . In agreement with previous findings, the results of the present study indicated that the effects of estrogen can be exerted directly through the ER in monocytes/macrophages (27). This is also in agreement with another report demonstrating that the expression and phosphorylation level of TAK1 significantly decreased following treatment with estradiol in PC12 cells (28).

A previous study reported that treatment with E2 significantly inhibited experimental autoimmune arthritis (29). However, the mechanism underlying these effects has yet to be fully elucidated. The data of the present study demonstrated that treatment with E2 decreased the incidence and severity of CIA and also decreased the expression of TAK1. However, the molecular mechanisms underlying the role of $\mathrm{E} 2$ in regulating the expression of TAK 1 must be further investigated in future studies.

In summary, the results of the present study uncovered a previously unidentified role of E2 in regulating TAK1 expression and suppressing the pathological process of CIA. Thus, TAK1 may serve as a potential diagnostic and therapeutic target for the treatment of RA.

\section{Acknowledgements}

Not applicable.

\section{Funding}

The present study was funded by the National Natural Science Fund Project (grant no. 81401334), the Natural Science Fund Projects of Liaoning Province (grant no. 20170540543) and the Scientific Project from Liaoning Education Department (grant no. LQNK201730).

\section{Availability of data and materials}

All data generated or analyzed during the present study are included in this published article.

\section{Authors' contributions}

XL collected clinical tissues and conducted the experiments. ML designed the study and wrote the manuscript. Both authors approved the final version of the manuscript.

\section{Ethics approval and consent to participate}

The present study was approved by the Ethics Committee of China Medical University (Shenyang, China). Written informed consent was obtained from all participants.

\section{Patient consent for publication}

All participants provided written informed consent for publication.

\section{Competing interests}

The authors declare that they have no competing interests.

\section{References}

1. Viatte S, Plant D and Raychaudhuri S: Genetics and epigenetics of rheumatoid arthritis. Nat Rev Rheumatol 9: 141-153, 2013.

2. Juarez M, Filer A and Buckley CD: Fibroblasts as therapeutic targets in rheumatoid arthritis and cancer. Swiss Med Wkly 142: w13529, 2012.

3. Huh JE, Seo BK, Park YC, Kim JI, Lee JD, Choi DY, Baek YH and Park DS: WIN-34B, a new herbal medicine, inhibits the inflammatory response by inactivating $\mathrm{I} \kappa \mathrm{B}-\alpha$ phosphorylation and mitogen activated protein kinase pathways in fibroblast-like synoviocytes. J Ethnopharmacol 143: 779-786, 2012.

4. Xu L, Feng X, Tan W, Gu W, Guo D, Zhang M and Wang F: IL-29 enhances Toll-like receptor-mediated IL-6 and IL-8 production by the synovial fibroblasts from rheumatoid arthritis patients. Arthritis Res Ther 15: R170, 2013.

5. Yoshioka Y, Kozawa E, Urakawa H, Arai E, Futamura N Zhuo L, Kimata K, Ishiguro N and Nishida Y: Suppression of hyaluronan synthesis alleviates inflammatory responses in murine arthritis and in human rheumatoid synovial fibroblasts. Arthritis Rheum 65: 1160-1170, 2013.

6. Hammaker DR, Boyle DL, Inoue T and Firestein GS: Regulation of the JNK pathway by TGF-beta activated kinase 1 in rheumatoid arthritis synoviocytes. Arthritis Res Ther 9: R57, 2007.

7. Choi YS, Park JK, Kang EH, Lee YK, Kim TK, Chung JH, Zimmerer JM, Carson WE III, Song YW and Lee YJ: Cytokine signaling-1 suppressor is inducible by IL-1beta and inhibits the catabolic effects of IL-1beta in chondrocytes: Its implication in the paradoxical joint-protective role of IL-1beta. Arthritis Res Ther 15: R191, 2013.

8. Geurts J, van den Brand BT, Wolf A, Abdollahi-Roodsaz S, Arntz OJ, Kracht M, van den Berg WB and van de Loo FA: Toll-like receptor 4 signalling is specifically TGF-beta-activated kinase 1 independent in synovial fibroblasts. Rheumatology (Oxford) 50: 1216-1225, 2011.

9. Alamanos Y and Drosos AA: Epidemiology of adult rheumatoid arthritis. Autoimmun Rev 4: 130-136, 2005.

10. Engdahl C, Jochems C, Windahl SH, Börjesson AE, Ohlsson C, Carlsten $\mathrm{H}$ and Lagerquist MK: Amelioration of collagen-induced arthritis and immune-associated bone loss through signaling via estrogen receptor alpha, and not estrogen receptor beta or G protein-coupled receptor 30. Arthritis Rheum 62: 524-533, 2010.

11. Holmdahl R, Carlsten H, Jansson L and Larsson P: Oestrogen is a potent immunomodulator of murine experimental rheumatoid disease. Br J Rheumatol 28 (Suppl 1): 54-58, discussion 69-71, 1989.

12. Arnett FC, Edworthy SM, Bloch DA, McShane DJ, Fries JF, Cooper NS, Healey LA, Kaplan SR, Liang MH, Luthra HS, et al: The American Rheumatism Association 1987 revised criteria for the classification of rheumatoid arthritis. Arthritis Rheum 31: 315-324, 1988 .

13. Villeneuve E, Nam J and Emery P: 2010 ACR-EULAR classification criteria for rheumatoid arthritis. Rev Bras Reumatol 50: 481-483, 2010.

14. Bartok B, Boyle DL, Liu Y, Ren P, Ball ST, Bugbee WD, Rommel C and Firestein GS: PI3 kinase $\delta$ is a key regulator of synoviocyte function in rheumatoid arthritis. Am J Pathol 180: 1906-1916, 2012.

15. Livak KJ and Schmittgen TD: Analysis of relative gene expression data using real-time quantitative PCR and the 2(- $\Delta \Delta$ C(T)) Method. Methods 25: 402-408, 2001

16. Kim JM, Shin SC, Park GC, Lee JC, Jeon YK, Ahn SJ, Thibeault S and Lee BJ: Effect of sex hormones on extracellular matrix of lamina propria in rat vocal fold. Laryngoscope 30: 732-740, 2020

17. Andersson A, Grahnemo L, Engdahl C, Stubelius A, Lagerquist MK, Carlsten $\mathrm{H}$ and Islander U: IL-17-producing $\gamma \delta \mathrm{T}$ cells are regulated by estrogen during development of experimental arthritis. Clin Immunol 161: 324-332, 2015.

18. Gottar-Guillier M, Dodeller F, Huesken D, Iourgenko V, Mickanin C, Labow M, Gaveriaux S, Kinzel B, Mueller M, Alitalo K, et al: The tyrosine kinase BMX is an essential mediator of inflammatory arthritis in a kinase-independent manner. J Immunol 186: 6014-6023, 2011. 
19. Tedeschi SK, Bermas B and Costenbader KH: Sexual disparities in the incidence and course of SLE and RA. Clin Immunol 149: 211-218, 2013.

20. Islander U, Jochems C, Lagerquist MK, Forsblad-d'Elia H and Carlsten H: Estrogens in rheumatoid arthritis; the immune system and bone. Mol Cell Endocrinol 335: 14-29, 2011.

21. Kvien TK, Uhlig T, Ødegård S and Heiberg MS: Epidemiological aspects of rheumatoid arthritis: The sex ratio. Ann N Y Acad Sci 1069: 212-222, 2006.

22. Khosla S, Melton LJ III, Atkinson EJ and O'Fallon WM: Relationship of serum sex steroid levels to longitudinal changes in bone density in young versus elderly men. J Clin Endocrinol Metab 86: 3555-3561, 2001

23. Jung SM, Kim KW, Yang $\mathrm{CW}$, Park $\mathrm{SH}$ and $\mathrm{Ju} \mathrm{JH}$ : Cytokine-mediated bone destruction in rheumatoid arthritis J Immunol Res 2014: 263625, 2014.

24. Cutolo M, Accardo S, Villaggio B, Clerico P, Bagnasco M, Coviello DA, Carruba G, lo Casto M and Castagnetta L: Presence of estrogen-binding sites on macrophage-like synoviocytes and $\mathrm{CD}^{+}, \mathrm{CD} 29^{+}, \mathrm{CD} 45 \mathrm{RO}^{+} \mathrm{T}$ lymphocytes in normal and rheumatoid synovium. Arthritis Rheum 36: 1087-1097, 1993.
25. Cutolo M, Capellino S, Montagna P, Sulli A, Seriolo B and Villaggio B: Anti-inflammatory effects of leflunomide in combination with methotrexate on co-culture of $\mathrm{T}$ lymphocytes and synovial macrophages from rheumatoid arthritis patients. Ann Rheum Dis 65: 728-735, 2006.

26. Dubey RK, Tofovic SP and Jackson EK: Cardiovascular pharmacology of estradiol metabolites. J Pharmacol Exp Ther 308: 403-409, 2004.

27. Cutolo M, Villaggio B, Bisso A, Sulli A, Coviello D and Dayer JM: Presence of estrogen receptors in human myeloid monocytic cells (THP-1 cell line). Eur Cytokine Netw 12: 368-372, 2001.

28. Peng R, Dai W and Li Y: Neuroprotective effect of a physiological ratio of testosterone and estradiol on corticosterone induced apoptosis in PC12 cells via Traf6/TAK1 pathway. Toxicol In Vitro 50: 257-263, 2018.

29. Andersson A, Stubelius A, Karlsson MN, Engdahl C, Erlandsson M, Grahnemo L, Lagerquist MK and Islander U: Estrogen regulates $\mathrm{T}$ helper 17 phenotype and localization in experimental autoimmune arthritis. Arthritis Res Ther 17: 32, 2015. 